\title{
Avaliação do Risco de Recidiva de Escurecimento Após Tratamento Clareador Dental
}

\author{
Assessment of the Risk of Recurrence of darkening After Tooth Whitening Treatment
}

\author{
Alípio Pinto Pereira Guedes*a; Artur José Carreira ${ }^{\text {a }}$ Paulo Henrique Perlatti D’Alpino ${ }^{\mathrm{a}}$
}

aUniversidade Anhanguera de São Paulo, Programa de Pós-Graduação Stricto Sensu em Biotecnologia e Inovação em Saúde. SP, Brasil.

*E-mail: alipioppguedes@gmail.com

\begin{abstract}
Resumo
Na busca por um sorriso mais harmônico, pacientes tem escolhido o tratamento clareador dental por ser uma técnica considerada não invasiva e efetiva. Porém há uma dificuldade dos profissionais em oferecer aos pacientes um prognóstico do tratamento clareador dental, especialmente quanto a prazos de retorno para avaliação e até mesmo de orientação quanto ao risco de recidiva do escurecimento individual. Essas orientações e tomadas de decisões são feitas geralmente de forma subjetiva e sem critérios previamente estabelecidos. O prognóstico do tratamento e estabelecimento de retornos para reavaliação devem levar em consideração alguns hábitos deletérios individuais que podem levar à pigmentação dos dentes. O presente estudo propôs uma individualização no tratamento clareador dental que inclui a avaliação do risco de recidiva de escurecimento dos dentes após tratamento clareador. Propôs-se um questionário contendo perguntas objetivas em relação a alguns hábitos (como tabagismo, café, hábitos de higiene) a partir do qual se estabelece uma pontuação. Por meio deste instrumento, é possível estabelecer o risco de recidiva de escurecimento e também o período de retorno para reavaliação através de uma abordagem simples e objetiva por meio de escores. O uso do questionário aqui proposto deve fazer parte da anamnese, possibilitando assim um melhor prognóstico e acompanhamento da manutenção dos tratamentos clareadores e redução de forma eficaz os riscos desnecessários de tratamentos repetitivos em prazos de retorno muito curtos.
\end{abstract}

Palavras-chave: Clareamento Dental. Prognóstico. Assistência Odontológica. Anamnese.

\begin{abstract}
When searching for a more harmonious smile, patients have chosen dental bleaching treatment for being considered a non-invasive and effective technique. However, clinicians are unsure to provide patients a prognosis for dental bleaching, especially to predict the time to which patients should return for reevaluation and even a guidance to predict the risk of individual darkening. These orientations and decisionmaking in the management of recalls are generally subjective and without previously established criteria. The prognosis of treatment and establishment of recalls should take into account deleterious individual habits that can lead to pigmentation of the teeth. The present study proposed individualization in the dental bleaching treatment that includes the assessment of the risk of recurrence of darkening after treatment. A questionnaire was proposed with objective questions about personal habits (such as smoking, coffee, and oral care). With this simple and objective scoring method, it is possible to determine the risk of recurrence of darkening, and also, the recall intervals. The questionnaire proposed here should be part of the anamnesis, thus enabling a better prognosis and monitoring of the recall frequency of dental bleaching treatments, effectively reducing the unnecessary risks of repetitive treatments in short return times.
\end{abstract}

Keywords: Tooth Bleaching. Prognosis. Dental Care. Medical History Taking.

\section{Introdução}

A necessidade da melhoria e manutenção da estética dos dentes e sua cor são temas importantes atualmente, seja para cirurgiões-dentistas que desejam determinar corretamente o material restaurador estético para maximizar a recriação da estrutura natural do dente, para técnicos em odontologia que visam replicar a forma e a qualidade da aparência do dente e, finalmente para a população em geral que deseja aprimorar seus sorrisos (WATTS; ADDY, 2001; OKUDA, 2013). Dentro de um planejamento e prognóstico dos tratamentos odontológicos disponíveis, sejam restauradores ou preventivos, a cor dos dentes dos pacientes vem tomando cada vez mais um lugar de destaque como queixa principal e até como parte de um tratamento prévio dentro do plano de tratamento (EPPLE et al, 2019).
Sabe-se que cor dos dentes é influenciada por uma combinação de sua cor intrínseca e pela presença de quaisquer manchas extrínsecas que possam se formar na superfície e na profundidade do esmalte dental, o que gera uma insatisfação quanto à coloração dos dentes (JOINER; LUO, 2017). Em geral, essa insatisfação com a cor dos dentes mostrouse associada ao aumento do desejo de tratamentos que melhoram a estética dental, incluindo o clareamento dos dentes (SAMORODNITZKY-NAVEH et al., 2007). De fato, o mercado de clareamento dental cresceu significativamente com uma ampla variedade de abordagens e produtos para clareamento dental, incluindo cremes dentais e enxaguatórios bucais, dentre outras formas (CAREY, 2014). A busca por dentes brancos por meio de tratamentos clareadores está relacionado ao fato de que o branco, de forma geral, está relacionado à cor da pureza, 
frescura e limpeza, sendo uma combinação de todas as cores do espectro da luz visível (JOINER; LUO, 2017).

Apesar de estudos recentes mostrarem a eficácia de diferentes técnicas de clareamento dental, pode haver a possibilidade do aparecimento de novas manchas após tratamentos clareadores em curto prazo (FERNANDEZ et al., 2017). O reaparecimento de novas manchas é decorrente muitas vezes de tratamentos não controlados pelos pacientes e a reexposição a outros hábitos alimentares e cosméticos (KARADA; SEVEN, 2014). Os estudos não são conclusivos em apontar a causa da remissão de manchas intrínsecas ou extrínsecas após o tratamento clareador, sendo apontadas possivelmente diferenças de protocolos clínicos, produtos utilizados, tipo de agente clareador e, ainda, as concentrações destes agentes MATIS et al., 2000). Outro fator a se destacar é o fato de que o mecanismo de ação do clareamento dental por peróxido de hidrogênio não é completamente conhecido (ASCHHEIM, 2015). Uma eventual alteração da coloração dos dentes constitui uma desarmonia que é imediatamente notada, sendo um fator isolado importante causando desequilíbrio estético do sorriso. Na busca por um sorriso mais harmônico tem-se utilizado o tratamento clareador dental por ser uma técnica considerada não invasiva e efetiva desde que os materiais utilizados sejam adequadamente selecionados e empregados (REZENDE et al., 2013).

Na clínica diária há dificuldade por parte dos profissionais em estabelecer um prognóstico do tratamento clareador aos pacientes, de se estabelecer prazos para retorno para avaliação e, até mesmo, para orientar os pacientes quanto aos riscos de escurecimento dental pós-tratamento clareador. Geralmente estas orientações e tomadas de decisões são feitas de forma subjetiva e sem critérios definidos que levem em consideração certos hábitos deletérios aos dentes que causam escurecimento do esmalte (TAY et al., 2012). Partindo desta premissa, e em função da falta de uma diretriz mais precisa dentro do tratamento clareador dental, e até da necessidade do estabelecimento de um prognóstico deste tratamento, observou-se que havia a necessidade da avaliação do paciente quanto a hábitos comuns para uma correta avaliação do caso e de seu acompanhamento.

O objetivo do presente estudo é propor uma avaliação clínica do risco de recidiva do escurecimento após tratamento clareador dental, por meio de um método de pontuação (escores) simples e específico em função dos hábitos dos pacientes. Com base em um instrumento específico, propõese a aplicação de um questionário estruturado durante a anamnese para se determinar, com base na pontuação obtida, o risco de recorrência do escurecimento e também determinar intervalos de reavaliação pós-tratamento. Levando em conta que o tratamento clareador dental não é livre de riscos e que novas intervenções para manutenção podem ser necessárias, espera-se que este sistema de pontuação forneça subsídios aos clínicos para escolha do tratamento clareador, bem como indicadores de previsibilidade para que possa reduzir de forma eficaz os riscos desnecessários de tratamentos repetitivos em prazos de retorno muito curtos.

\section{Material e Métodos}

Desenvolveu-se então uma anamnese específica para o risco de escurecimento dental, por meio de uma abordagem objetiva e simples dos hábitos pessoais do paciente. Os itens selecionados relativos aos hábitos dos pacientes foram baseados em referências que se tem na literatura sobre as causas extrínsecas do escurecimento dental (FERNANDEZ et al., 2017; FERRAZ et al., 2019), podendo e devendo ser adaptadas a realidades regionais. O Quadro 1 apresenta um questionário como parte da anamnese que contempla perguntas em relação à 5 hábitos onde são abordados o seu uso e frequência diária. Os questionamentos desses cinco hábitos são analisados de forma objetiva e direta com perguntas de resposta simples direcionadas ao paciente que se submeterá ao tratamento.

Quadro 1 - Anamnese de avaliação do risco de recidiva de escurecimento após tratamento clareador dental em função dos hábitos do paciente

\begin{tabular}{|c|c|}
\hline \multicolumn{2}{|c|}{$\begin{array}{c}\text { Pontuações para determinação do risco de recidiva de } \\
\text { escurecimento }\end{array}$} \\
\hline 1) Escovacão & \\
\hline Não & $(15)$ \\
\hline Sim & $(1)$ \\
\hline \multicolumn{2}{|c|}{ Se sim, qual a frequência diária? } \\
\hline - 1 vez ao dia & $(10)$ \\
\hline - 2 vezes ao dia & $(5)$ \\
\hline - 3 vezes ao dia ou mais & $(1)$ \\
\hline \multicolumn{2}{|l|}{ 2) Fio dental } \\
\hline Não & $(10)$ \\
\hline Sim & $(1)$ \\
\hline \multicolumn{2}{|c|}{ Se sim, qual a frequência diária? } \\
\hline - Diariamente & $(1)$ \\
\hline - De vez em quando & $(5)$ \\
\hline \multicolumn{2}{|l|}{ 3) Café } \\
\hline Não & $(1)$ \\
\hline Sim & $(15)$ \\
\hline \multicolumn{2}{|c|}{ Se sim, qual a frequência diária? } \\
\hline - 1 a 2 vezes ao dia & $(5)$ \\
\hline - $\quad$ Mais que 3 vezes ao dia & $(10)$ \\
\hline \multicolumn{2}{|l|}{ 4) Molhos escuros } \\
\hline Não & $(1)$ \\
\hline Sim & $(5)$ \\
\hline \multicolumn{2}{|c|}{ Se sim, qual a frequência diária? } \\
\hline - Diariamente & $(10)$ \\
\hline - Às vezes & $(5)$ \\
\hline \multicolumn{2}{|l|}{ 5) Fumo } \\
\hline Não & $(1)$ \\
\hline Sim & $(20)$ \\
\hline \multicolumn{2}{|c|}{ Se sim, qual a frequência diária? } \\
\hline - Até 10 cigarros & $(5)$ \\
\hline - 10 a 20 cigarros & $(10)$ \\
\hline - Mais que 20 cigarros & $(15)$ \\
\hline
\end{tabular}


Foram elencadas as pontuações em função de cada um dos itens e subitens previamente determinados (Quadro 1), sendo que a partir desses valores obtemos uma pontuação que avalia o risco de recidiva do escurecimento após tratamento clareador por meio de uma pontuação fundamentada no questionário desta anamnese, que deve nortear o tratamento, direcionando a necessidade de retorno em função de uma determinada pontuação, para reavaliação periódica (Quadro 2).

Quadro 2 - Determinação do risco de escurecimento dental em função da pontuação obtida

\begin{tabular}{|ll|}
\hline \multicolumn{1}{|c|}{ Risco de escurecimento dental } \\
\hline$>$ & Até 10: Baixíssimo risco \\
\hline$>$ & 11 Até 30: Baixo risco \\
\hline$>$ & 31 a 50: Médio risco \\
\hline$>$ & 51 a70: Alto risco \\
\hline$>$ & Acima de 71: Altíssimo risco \\
\hline
\end{tabular}

Fonte: Dados da pesquisa.

Os clínicos podem lançar mão do instrumento que está no Quadro 1 que pode ser utilizado que discrimina os riscos de recidiva do escurecimento dos dentes após tratamento clareador, é possível determinar o tempo ideal para retorno para que um determinado paciente seja reavaliado quanto à sua satisfação quanto à coloração de seus dentes e a necessidade ou não de um novo tratamento clareador dental, descrito no Quadro 3.

Quadro 3 - Programação de retornos para avaliação do tratamento clareador em função do risco avaliado

\begin{tabular}{|ll|}
\hline \multicolumn{1}{|c|}{ Programação de retorno } \\
\hline$>$ & Baixíssimo risco: retorno após 24 meses \\
\hline$>$ & Baixo risco: retorno após 18 meses \\
\hline$>$ & Médio risco: retorno após 12 meses \\
\hline$>$ & Alto risco: retorno após 6 meses \\
\hline$>$ & Altíssimo risco: retorno após 3 meses \\
\hline
\end{tabular}

Fonte: Dados da pesquisa.

Esse retorno deve ser enfatizado ao paciente que se relaciona ao tratamento clareador e não às condições gerais da saúde bucal, variando de 3 a 24 meses, dependendo do risco individual (Quadro 2).

\section{Resultados e Discussão}

Uma das dúvidas mais frequentes dos pacientes candidatos ao tratamento clareador dental é quanto à necessidade de se repetir o procedimento e quando fazê-lo. Com uma anamnese específica e objetiva para o risco de recidiva de escurecimento dental após o tratamento clareador, o profissional pode individualizar o acompanhamento de cada caso, permitindo uma atenção maior no pós-tratamento e uma maior fidelização dos pacientes. As causas das manchas nos dentes são muito variadas em cada paciente, por isso é muito importante estudá-las para um melhor diagnóstico e poder selecionar o tratamento clareador mais adequado (STRASSLER, 2006). É importante destacar que o compromisso do paciente em relação à manutenção do tratamento é necessário para obter ótimos resultados, mesmo que em longo prazo (BRUZELL et al., 2013). O tratamento clareador dental quando realizado de forma adequada, levando-se em conta bases científicas para sua indicação e fazendo-se o uso correto dos produtos, vem de encontro a uma odontologia minimamente invasiva que devolve e promove uma estética do sorriso (PETER et al., 2007). Dentro de um planejamento estético hoje temos o tratamento clareador como primeira escolha e etapa para obter um sorriso equilibrado (JOINER; LUO, 2017).

Uma superfície branca, em termos físicos, é aquela que reflete fortemente (mais de 50\%) em todo o espectro visível (HUNTER, 1987). A cor intrínseca de um dente é determinada pela maneira como a luz é dispersa e absorvida na superfície e nas estruturas do dente (TEN BOSCH; COOPS, 1995). Os produtos indicados para clareamento dental geralmente ajudam a melhorar a aparência geral dos dentes, seja alterando sua cor intrínseca ou removendo e controlando a formação de manchas extrínsecas (JOINER; LUO, 2017). Estudos psicofísicos sobre a cor e a brancura dos dentes por meio da avaliação de imagens indicaram que os dentes clareados levam a julgamentos mais positivos sobre traços de personalidade, como competência e apelo social, capacidade intelectual e satisfação no relacionamento (KERSHAW et al., 2008).

Em geral, as manchas dos dentes são classificadas de acordo com sua localização em descolorações intrínsecas ou extrínsecas (JOINER; LUO, 2017). A cor intrínseca do dente está associada à dispersão da luz e propriedades de absorção do esmalte e dentina (TEN BOSCH; COOPS, 1995). Esse tipo de descoloração ocorre quando uma alteração na composição estrutural ou na espessura dos tecidos dentinários aparece durante o desenvolvimento dentário ou após erupção. Dentro das descolorações intrínsecas, existem vários subgrupos para uma classificação mais precisa (RODRIGUEZ-MARTINEZ et al., 2019). Vários distúrbios metabólicos promovem a descoloração dos dentes (WATANABE et al., 1999). A cor do dente também pode ser alterada por ação de alguns tratamentos médicos. Um grupo bem conhecido de antibióticos que causa descoloração intrínseca do dente são as tetraciclinas (SANCHESZ et al., 2004). Doenças hereditárias e idiopáticas também podem ser as causas da descoloração dos dentes (DA COSTA-SILVA et al., 2011). Outro subgrupo de descoloração são as causas traumáticas (SULIEMAN, 2005). Por outro lado, a cor extrínseca está associada à adsorção de diferentes compostos na superfície do esmalte, que podem desenvolver manchas extrínsecas (WITKOP, 1988). Essas descolorações são causadas por substâncias cromogênicas que aderem diretamente ao dente (coloração direta) ou por interação química com compostos externos com outro dente elemento de superfície (coloração indireta), este último geralmente está associada à presença de sais metálicos (THICKETT; COBOURNE, 2009).

A maioria dos produtos destinados a tratamentos clareadores contém peróxido de hidrogênio ou peróxido de carbamida, formulados geralmente na forma de géis, 
sendo aplicados nos dentes em vários formatos, incluindo protetores bucais, tiras ou mesmo de aplicação direta (JOINER; LUO, 2017). O peróxido difunde-se na intimidade do esmalte dental, onde descolora ou "quebra" compostos orgânicos em partículas menores, e como consequência há o "branqueamento" dos dentes (KWON; WERTZ, 2015). Inúmeros sistemas clareadores foram desenvolvidos, sendo classificados em função de diferentes parâmetros como: tipo de agente clareador, concentração, forma de aplicação ou periodicidade (JOINER; LUO, 2017). De forma geral, os sistemas de clareamento são divididos em dois grandes grupos: sistemas de clareamento em consultório e caseiro (HEYMANN, 2005).

Apesar de todo o conhecimento sobre clareamento dental, podem ocorrer efeitos colaterais associados ao uso de diferentes produtos para clareamento (DAHL; PALLESEN, 2003). Os problemas mais comuns são os efeitos locais, podendo causar alterações nos tecidos dentários e na mucosa oral (RODRIGUEZ-MARTINEZ et al., 2019). Essas questões podem também causar efeitos indesejáveis, como hipersensibilidade dentária, reabsorção radicular, modificações na morfologia da superfície do esmalte dental, irritação gengival e efeitos corrosivos superficiais em restaurações (GOLDBERG et al., 2010). Ainda é necessário desenvolver um método mais eficaz, eficiente, e tratamento duradouro para obter um efeito de clareamento ideal sem nenhum efeito colateral (RODRIGUEZ-MARTINEZ et al., 2019).

Idealmente, sugere-se que um protocolo de um tratamento clareador dental deveria divide-se em procedimentos pré, trans e pós-tratamento. Desta forma, individualizandose o tratamento e acompanhando o paciente incluindose o pós-operatório, os possíveis efeitos adversos podem ser minimizados. Orientações quanto à importância da necessidade de restrições alimentares, principalmente os que possuem alto poder corante, são comumente passadas para os pacientes. É fato que o consumo de bebidas como chás, cafés, refrigerantes e, também, o tabagismo podem resultar no manchamento da superfície dental (KARADAS; SEVEN, 2014). O uso constante desses alimentos pode, ao longo do tempo, pigmentar a superfície do esmalte, mas a restrição de alimentos por parte dos pacientes além de ser de difícil adesão pelos mesmos, ainda é um assunto bastante controverso (MORIMOTO et al., 2018).

O sucesso do tratamento clareador dental inicia-se na realização de uma anamnese criteriosa para se conhecer a saúde e expectativas do paciente. Nesta fase é importante sanar todas as dúvidas do paciente quanto ao tratamento, informando o prognóstico, efeitos adversos que podem ocorrer e os limites que o caso apresenta. $\mathrm{O}$ paciente deve ser alertado sobre o seu risco de escurecimento dental, podendo e devendo ser orientado quanto aos hábitos que podem ser alterados para se ter uma melhor estabilidade do resultado final do tratamento. Sendo assim, espera-se que com o uso da ferramenta proposta no presente estudo, seja possível realizar uma anamnese para avaliação do risco de escurecimento dental após tratamento clareador, avaliando-se e enquadrando o paciente num plano de tratamento e retornos. Sendo assim, a cada retorno para avaliação, esta ferramenta possibilita reenquadrar o paciente no plano de tratamento individualizado, que favorecem o acompanhamento mais adequado. A aplicação da anamnese de risco de escurecimento dental e com a programação dos retornos, melhoram a confiança e fidelização ao profissional, oferecendo ao paciente um pós-tratamento adequado com um melhor controle da estabilidade do resultado, da valorização do tratamento pelo paciente e da importância deste com os hábitos alimentares e de higiene bucal. Estudos clínicos são ainda necessários para avaliar e validar o sistema de pontuação proposto para a avaliação do risco de recidiva de escurecimento após tratamento clareador dental.

\section{Conclusão}

Conclui-se que o questionário proposto no presente estudo pode ser um importante instrumento para auxiliar no prognóstico de tratamentos clareadores dentais, fazendo parte da anamnese. Por meio de uma pontuação (escores) baseada nos hábitos do paciente, pode-se determinar o risco de recorrência de escurecimento após tratamento clareador dental através de um questionário estruturado simples e específico. Além disso, este instrumento também auxilia os clínicos a determinarem os intervalos de reavaliação pós-tratamento, favorecendo um melhor acompanhamento da manutenção dos resultados obtidos e evolução do paciente frente ao tratamento clareador.

\section{Referências}

ASCHHEIM, K.W. Bleaching and related agents. New York: CV Mosby, 2015.

BRUZELL, E.M. et al. Side effects of external tooth bleaching: a multi-centre practice-based prospective study. Bras. Dent. J., v. 215, n. 9, p. E17-E17, 2013. doi: 10.1038/sj.bdj.2013.1047.

CAREY, C. M. Tooth whitening: what we now know. J. Evid. Based Dent. Pract., v.14, p.70-76, 2014. doi: 10.1016/j. jebdp.2014.02.006.

DA COSTA-SILVA, C. M. et al. Increase in severity of molarincisor hypomineralization and its relationship with the colour of enamel opacity: a prospective cohort study. Int. J. Paediatr. Dent., v.21, n.5, p. 333-41, 2011. doi: 10.1111/j.1365263X.2011.01128.x.

DAHL, J. E.; PALLESEN, U. Tooth bleaching--a critical review of the biological aspects. Crit Rev. Oral Biol. Med., v.14, n.4, p.292-304, 2003. doi: 10.1177/154411130301400406.

EPPLE, M. et al. A critical review of modern concepts for teeth whitening. Dent. J. (Basel), v.7, n.3, p.79, 2019. doi: 10.3390/ dj7030079.

FERNANDEZ, E. et al. Longevity, esthetic perception, and psychosocial impact of teeth bleaching by low $(6 \%)$ hydrogen peroxide concentration for in-office treatment: a randomized clinical trial. Oper. Dent., v.42, n.1, p.41-52, 2017. doi: 10.2341/15-335-C. 
FERRAZ, N. K. L. et al. Longevity, effectiveness, safety, and impact on quality of life of low-concentration hydrogen peroxides in-office bleaching: a randomized clinical trial. Clin. Oral Investig., v.23, n.5, p.2061-2070, 2019. doi: 10.1007/ s00784-018-2607-7.

GOLDBERG, M. et al. Undesirable and adverse effects of toothwhitening products: a review. Clin. Oral. Investig., v.14, n.1, p.110, 2010. doi: 10.1007/s00784-009-0302-4.

HEYMANN, H. O. Tooth whitening: facts and fallacies. Bras. Dent. J., v.198, n.8, p.514, 2005. doi: 10.1038/sj.bdj.4812298.

HUNTER, R.S. The measurement of appearance. New Youk: John Wiley \& Sons, 1987.

JOINER, A.; LUO, W. Tooth colour and whiteness: a review. $J$. Dent., v.67S, p.S3-S10, 2017. doi: 10.1016/j.jdent.2017.09.006.

KARADAS, M.; SEVEN, N. The effect of different drinks on tooth color after home bleaching. Euro. J. Dent., v.8, n.2, p.249253, 2014. doi: 10.4103/1305-7456.130622.

KERSHAW, S. et al. The influence of tooth colour on the perceptions of personal characteristics among female dental patients: comparisons of unmodified, decayed and 'whitened' teeth. Bras. Dent. J., v.204, n.5, p.256-257, 2008. doi: 10.1038/ bdj.2008.134.

KWON, S.R.; WERTZ, P. W. Review of the mechanism of tooth whitening. J. Esthet. Restor. Dent., v.27, n.5, p.240-57, 2015. doi: 10.1111/jerd.12152.

MATIS, B.A. et al. Clinical evaluation of bleaching agents of different concentrations. Quintessence Int., v.31, p.303-310, 2000. doi:

MORIMOTO, S. et al. Effect of bleaching agents on black tooth stains caused by chromogenic bacteria: 10 years follow-up case report. Rev. Gaúcha Odontol., v.66, p.187-193, 2018. doi: 10.1590/1981-8637201800020000143457.

OKUDA, W.H. Minimally invasive dentistry and its impact on esthetic restorative dentistry. Gen. Dent., v.61, n.5, p.24-26, 2013. doi:

PETER, J. N. et al. Conservative aesthetic techniques for discoloured teeth: 2. Micro-abrasion and composite. Dent Update, v.34, n.3, p.160-166, 2007. doi: 10.12968/denu.2007.34.3.160.
REZENDE, M. et al. Clinical effects of exposure to coffee during at-home vital bleaching. Oper Dent., v.38, n.6, p.E229-236, 2013. doi: $10.2341 / 12-188-C$.

RODRIGUEZ-MARTINEZ, J. et al. Tooth whitening: From the established treatments to novel approaches to prevent side effects. J. Esthet. Restor. Dent., v.31, n.5, p.431-440, 2019. doi: 10.1111/ jerd.12519.

SAMORODNITZKY-NAVEH, G. R. et al. Patients' satisfaction with dental esthetics. J. Am. Dent. Assoc., v.138, n.6, p.805-8, 2007. doi: 10.14219/jada.archive.2007.0269.

SANCHEZ, A. R. et al. Tetracycline and other tetracyclinederivative staining of the teeth and oral cavity. Int. J. Dermatol., v.43, n.10, p.709-715, 2004. doi: 10.1111/j.13654632.2004.02108.x.

STRASSLER, H. E. Vital tooth bleaching: an update. Cont. Educ. Insert., v.4, p.1-8, 2006. doi:

SULIEMAN, M. An overview of tooth discoloration: extrinsic, intrinsic and internalized stains. Dent. Update, v.32, n.8, 2005. doi: 10.12968/denu.2005.32.8.463.

TAY, L. Y. et al. Long-term efficacy of in-office and at-home bleaching: a 2-year double-blind randomized clinical trial. Am. J. Dent., v.25, n.4, p.199-204, 2012. doi:

TEN BOSCH, J. J.; COOPS, J. C. Tooth color and reflectance as related to light scattering and enamel hardness. J. Dent. Res., v.74, n.1, p.374-380, 1995. doi: 10.1177/00220345950740011401.

THICKETT, E.; COBOURNE, M. T. New developments in tooth whitening. The current status of external bleaching in orthodontics. J. Orthod., v.36, n.3, p.194-201, 2009. doi: $10.1179 / 14653120723184$.

WATANABE, K. et al. Bilirubin pigmentation of human teeth caused by hyperbilirubinemia. J. Oral. Pathol. Med., v.28, n.3, p.128-130, 1999. doi: 10.1111/j.1600-0714.1999.tb02010.x.

WATTS, A.; ADDY, M. Tooth discolouration and staining: a review of the literature. Bras. Dent. J., v.190, n.6, p.309-16, 2001. doi: $10.1038 /$ sj.bdj.4800959.

WITKOP, C. J., JR. Amelogenesis imperfecta, dentinogenesis imperfecta and dentin dysplasia revisited: problems in classification. J. Oral. Pathol., v.17, n.9/10, p.547-53, 1988. doi: 10.1111/j.1600-0714.1988.tb01332. 